\title{
Boundedness of High Order Commutators of Riesz Transforms Associated with Schrödinger Type Operators
}

\author{
Yueshan Wang* \\ Department of Mathematics, Jiaozuo University, Jiaozuo 454003, Henan, China
}

Received 27 August 2017; Accepted (in revised version) 8 June 2018

\begin{abstract}
Let $\mathcal{L}_{2}=(-\Delta)^{2}+V^{2}$ be the Schrödinger type operator, where $V \neq 0$ is a nonnegative potential and belongs to the reverse Hölder class $R H_{q_{1}}$ for $q_{1}>n / 2, n \geq$ 5. The higher Riesz transform associated with $\mathcal{L}_{2}$ is denoted by $\mathcal{R}=\nabla^{2} \mathcal{L}_{2}^{-\frac{1}{2}}$ and its dual is denoted by $\mathcal{R}^{*}=\mathcal{L}_{2}^{-\frac{1}{2}} \nabla^{2}$. In this paper, we consider the $m$-order commutators $\left[b^{m}, \mathcal{R}\right]$ and $\left[b^{m}, \mathcal{R}^{*}\right]$, and establish the $\left(L^{p}, L^{q}\right)$-boundedness of these commutators when $b$ belongs to the new Campanato space $\Lambda_{\beta}^{\theta}(\rho)$ and $1 / q=1 / p-m \beta / n$.
\end{abstract}

Key Words: Schrödinger operator, Campanato space, Riesz transform, commutator.

AMS Subject Classifications: 42B25, 35J10, 42B35

\section{Introduction}

In this paper, we consider the Schrödinger type operator

$$
\mathcal{L}_{2}=(-\Delta)^{2}+V^{2} \text { on } \mathbb{R}^{n}, \quad n \geq 5,
$$

where $V$ is nonnegative, $V \neq 0$, and belongs to the reverse Hölder class $R H_{q}$ for some $q \geq n / 2$, i.e., there exists a constant $C$ such that

$$
\left(\frac{1}{|B|} \int_{B} V(y)^{q} d y\right)^{1 / q} \leq \frac{C}{|B|} \int_{B} V(y) d y
$$

for every ball $B \subset \mathbb{R}^{n}$.

The higher Riesz transform associated with $\mathcal{L}_{2}$ is defined by $\mathcal{R}=\nabla^{2} \mathcal{L}_{2}^{-1 / 2}$, and its dual is defined by $\mathcal{R}^{*}=\mathcal{L}_{2}^{-1 / 2} \nabla^{2}$. The $L^{p}$-boundedness of the higher Riesz transforms

*Corresponding author. Email address: wangys1962@163.com (Y. S. Wang) 
have been obtained in [1] by Liu and Dong: Suppose $V \in R H_{q_{1}}$ with $n / 2<q_{1}<n$. Let $1 / p_{1}=2 / q_{1}-2 / n, p_{1}^{\prime}=p_{1} /\left(p_{1}-1\right)$. If $1<p<p_{1}$, then for all $f \in L^{p}\left(\mathbb{R}^{n}\right)$,

$$
\|\mathcal{R} f\|_{L^{p}\left(\mathbb{R}^{n}\right)} \leq C\|f\|_{L^{p}\left(\mathbb{R}^{n}\right)} .
$$

If $p_{1}^{\prime}<p<\infty$, then for all $f \in L^{p}\left(\mathbb{R}^{n}\right)$,

$$
\left\|\mathcal{R}^{*} f\right\|_{L^{p}\left(\mathbb{R}^{n}\right)} \leq C\|f\|_{L^{p}\left(\mathbb{R}^{n}\right)} .
$$

As in [2], for a given potential $V \in R H_{q}$ with $q>n / 2$, we define the auxiliary function

$$
\rho(x)=\sup \left\{r>0: \frac{1}{r^{n-2}} \int_{B(x, r)} V(y) d y \leq 1\right\}, \quad x \in \mathbb{R}^{n} .
$$

It is well known that $0<\rho(x)<\infty$ for any $x \in \mathbb{R}^{n}$.

Let $\theta>0$ and $0<\beta<1$, in view of [3], the new Campanato class $\Lambda_{\beta}^{\theta}(\rho)$ consists of the locally integrable functions $b$ such that

$$
\frac{1}{|B(x, r)|^{1+\beta / n}} \int_{B(x, r)}\left|b(y)-b_{B}\right| d y \leq C\left(1+\frac{r}{\rho(x)}\right)^{\theta}
$$

for all $x \in \mathbb{R}^{n}$ and $r>0$. A seminorm of $b \in \Lambda_{\beta}^{\theta}(\rho)$, denoted by $[b]_{\beta}^{\theta}$, is given by the infimum of the constants in the inequalities above.

Note that if $\theta=0, \Lambda_{\beta}^{\theta}(\rho)$ is the classical Campanato space; If $\beta=0, \Lambda_{\beta}^{\theta}(\rho)$ is exactly the space $B M O_{\theta}(\rho)$ introduced in [4].

We denote by $\mathcal{K}$ and $\mathcal{K}^{*}$ the kernels of $\mathcal{R}$ and $\mathcal{R}^{*}$, respectively. Let $b$ be a locally integrable function, $m$ be a positive integer. The $m$-order commutators generated by higher Riesz transform and $b$ are defined by

$$
\left[b^{m}, \mathcal{R}\right] f(x)=\int_{R^{n}} \mathcal{K}(x, y)(b(x)-b(y))^{m} f(y) d y
$$

and

$$
\left[b^{m}, \mathcal{R}^{*}\right] f(x)=\int_{R^{n}} \mathcal{K}^{*}(x, y)(b(x)-b(y))^{m} f(y) d y .
$$

In this paper, we are interested in the boundedness of $\left[b^{m}, \mathcal{R}\right]$ and $\left[b^{m}, \mathcal{R}^{*}\right]$ on Lebesgue space when $b$ belongs to the new Campanato class $\Lambda_{\beta}^{\theta}(\rho)$. The main result of this paper is as follows.

Theorem 1.1. Suppose $V \in R H_{q_{1}}$ with $n / 2<q_{1}<n, 1 / p_{1}=2 / q_{1}-2 / n, p_{1}^{\prime}=p_{1} /\left(p_{1}-1\right)$. Let $0<\beta<1$, and let $b \in \Lambda_{\beta}^{\theta}(\rho)$. If $p_{1}^{\prime}<p<\infty$, then for all $f \in L^{p}\left(\mathbb{R}^{n}\right)$,

$$
\left\|\left[b^{m}, \mathcal{R}^{*}\right] f\right\|_{L^{q}\left(\mathbb{R}^{n}\right)} \leq C\left([b]_{\beta}^{\theta}\right)^{m}\|f\|_{L^{p}\left(\mathbb{R}^{n}\right)}
$$

where $1 / q=1 / p-m \beta / n$. 
We immediately deduce the following result by duality.

Corollary 1.1. Suppose $V \in R H_{q_{1}}$ with $n / 2<q_{1}<n, \frac{1}{p_{1}}=\frac{2}{q_{1}}-\frac{2}{n}$. Let $0<\beta<1$, and let $b \in \Lambda_{\beta}^{\theta}(\rho)$. If $1<p<p_{1}$, then for all $f \in L^{p}\left(\mathbb{R}^{n}\right)$,

$$
\left\|\left[b^{m}, \mathcal{R}\right] f\right\|_{L^{q}\left(\mathbb{R}^{n}\right)} \leq C\left([b]_{\beta}^{\theta}\right)^{m}\|f\|_{L^{p}\left(\mathbb{R}^{n}\right)},
$$

where $1 / q=1 / p-m \beta / n$.

We shall use the symbol $A \lesssim B$ to indicate that there exists a universal positive constant $C$, independent of all important parameters, such that $A \leq C B . A \approx B$ means that $A \lesssim B$ and $B \lesssim A$

\section{Some preliminaries}

We recall some important properties concerning the auxiliary function.

Proposition 2.1 ([2]). Let $V \in R H_{n / 2}$. For the function $\rho$ there exist $C$ and $k_{0} \geq 1$ such that

$$
C^{-1} \rho(x)\left(1+\frac{|x-y|}{\rho(x)}\right)^{-k_{0}} \leq \rho(y) \leq C \rho(x)\left(1+\frac{|x-y|}{\rho(x)}\right)^{\frac{k_{0}}{1+k_{0}}}
$$

for all $x, y \in \mathbb{R}^{n}$.

Assume that $Q=B\left(x_{0}, \rho\left(x_{0}\right)\right)$, for $x \in Q$, Proposition 2.1 tell us that $\rho(x) \approx \rho(y)$, if $|x-y|<C \rho(x)$.

Lemma 2.1 ([5]). Let $k \in \mathbb{N}$ and $x \in 2^{k+1} B\left(x_{0}, r\right) \backslash 2^{k} B\left(x_{0}, r\right)$. Then we have

$$
\frac{1}{\left(1+\frac{2^{k_{r}}}{\rho(x)}\right)^{N}} \lesssim \frac{1}{\left(1+\frac{2^{k_{r}}}{\rho\left(x_{0}\right)}\right)^{N /\left(k_{0}+1\right)}}
$$

Lemma 2.2 ([6]). Suppose $V \in R H_{q_{1}}, q_{1} \geq n / 2$. Then there exists a constants $l_{0}>0$, such that

$$
\frac{1}{r^{n-2}} \int_{B(x, r)} V(y) d y \lesssim\left(1+\frac{r}{\rho(x)}\right)^{l_{0}} .
$$

The following finite overlapping property given by Dziubański and Zienkiewicz in [7].

Proposition 2.2. There exists a sequence of points $\left\{x_{k}\right\}_{k=1}^{\infty}$ in $\mathbb{R}^{n}$, so that the family of critical balls $Q_{k}=B\left(x_{k}, \rho\left(x_{k}\right)\right), k \geq 1$, satisfies

(i) $\bigcup_{k} Q_{k}=\mathbb{R}^{n}$.

(ii) There exists $N=N(\rho)$ such that for every $k \in N$, $\operatorname{card}\left\{j: 4 Q_{j} \cap 4 Q_{k}\right\} \leq N$. 
For $\alpha>0, g \in L_{l o c}^{1}\left(\mathbb{R}^{n}\right)$ and $x \in \mathbb{R}^{n}$, we introduce the following maximal functions

$$
\begin{aligned}
& M_{\rho, \alpha} g(x)=\sup _{x \in B \in \mathcal{B}_{\rho, \alpha}} \frac{1}{|B|} \int_{B}|g(y)| d y, \\
& M_{\rho, \alpha}^{\sharp} g(x)=\sup _{x \in B \in \mathcal{B}_{\rho, \alpha}} \frac{1}{|B|} \int_{B}\left|g(y)-g_{B}\right| d y,
\end{aligned}
$$

where $\mathcal{B}_{\rho, \alpha}=\left\{B(z, r): z \in \mathbb{R}^{n}\right.$ and $\left.r \leq \alpha \rho(y)\right\}$.

We have the following Fefferman-Stein type inequality.

Proposition 2.3 ([4]). For $1<p<\infty$, there exist $\delta$ and $\gamma$ such that if $\left\{Q_{k}\right\}_{k=1}^{\infty}$ is a sequence of balls as in Proposition 2.2, then

$$
\int_{\mathbb{R}^{n}}\left|M_{\rho, \delta} g(x)\right|^{p} d x \lesssim \int_{\mathbb{R}^{n}}\left|M_{\rho, \gamma}^{\sharp} g(x)\right|^{p} d x+\sum_{k}\left|Q_{k}\right|\left(\frac{1}{\left|Q_{k}\right|} \int_{2 Q_{k}}|g|\right)^{p}
$$

for all $g \in L_{l o c}^{1}\left(\mathbb{R}^{n}\right)$.

We give an inequality for the function $b \in \Lambda_{\beta}^{\theta}(\rho)$.

Lemma 2.3 ([3]). Let $1 \leq s<\infty, b \in \Lambda_{\beta}^{\theta}(\rho), 0<\beta<1, k \in \mathbb{N}$, and $B=B(x, r)$. Then we have

$$
\left(\frac{1}{\left|2^{k} B\right|} \int_{2^{k} B}\left|b(y)-b_{B}\right|^{s} d y\right)^{1 / s} \lesssim[b]_{\beta}^{\theta}\left(2^{k} r\right)^{\beta}\left(1+\frac{2^{k} r}{\rho(x)}\right)^{\theta^{\prime}}
$$

where $\theta^{\prime}=\left(k_{0}+1\right) \theta$ and $k_{0}$ is the constant appearing in Proposition 2.1.

Let $K$ and $K^{*}$ be the kernels of the Riesz transform $\mathcal{R}$ and its dual operator $\mathcal{R}^{*}$, respectively. Then $K(x, z)=K^{*}(z, x)$ and we have the following estimates.

Lemma 2.4 ([8]). Suppose $V \in R H_{q_{1}}$ with $n / 2<q_{1}<n$.

(i) For every $N$, there exists a constant $C_{N}>0$ such that

$$
\left|\mathcal{K}^{*}(x, z)\right| \leq \frac{C_{N}\left(1+\frac{|x-z|}{\rho(x)}\right)^{-N}}{|x-z|^{n-2}}\left(\int_{B(z,|x-z| / 4)} \frac{V^{2}(u)}{|u-z|^{n-2}} d u+\frac{1}{|x-z|^{2}}\right) .
$$

(ii) For every $N$ and $0<\delta<\min \left\{1,2-n / q_{1}\right\}$, there exists a constant $C_{N}>0$ such that

$$
\begin{aligned}
& \left|\mathcal{K}^{*}(x, z)-\mathcal{K}^{*}(y, z)\right| \\
\leq & \frac{C_{N}|x-y|^{\delta}\left(1+\frac{|x-z|}{\rho(x)}\right)^{-N}}{|x-z|^{n-2+\delta}}\left(\int_{B(z,|x-z|)} \frac{V^{2}(u)}{|u-z|^{n-2}} d u+\frac{1}{|x-z|^{2}}\right),
\end{aligned}
$$

where $|x-y|<|x-z| / 16$. 


\section{Proof of Theorem 1.1}

To prove Theorem 1.1, we first prove the following lemmas.

Lemma 3.1. Suppose $V \in R H_{q_{1}}$ with $n / 2<q_{1}<n$. Let $1 / p_{1}=2 / q_{1}-2 / n$, and $b \in \Lambda_{\beta}^{\theta}(\rho)$. If $p_{1}^{\prime}<s<\infty$, then for all $f \in L_{\text {loc }}^{s}\left(\mathbb{R}^{n}\right)$ and every critical ball $Q=B\left(x_{0}, \rho\left(x_{0}\right)\right)$,

$$
\begin{aligned}
& \frac{1}{|Q|} \int_{Q}\left|\left[b^{m}, \mathcal{R}^{*}\right] f(y)\right| d y \\
\lesssim & \left([b]_{\beta}^{\theta}\right)^{m} \inf _{x \in Q} M_{m \beta, s}(f)(x)+\sum_{\gamma=0}^{m-1}\left([b]_{\beta}^{\theta}\right)^{m-\gamma} \inf _{x \in Q} M_{(m-\gamma) \beta, s}\left(\left[b^{\gamma}, \mathcal{R}^{*}\right] f\right)(x),
\end{aligned}
$$

where

$$
M_{m \beta, s}(f)(x)=\sup _{x \in B}\left(\frac{1}{|B|^{1-m \beta s / n}} \int_{B}|f(y)|^{s} d y\right)^{1 / s} .
$$

Proof. By binomial theorem, we have

$$
\begin{aligned}
(b(y)-b(z))^{m} & =\sum_{l=1}^{m} C_{l, m}(b(y)-\lambda)^{l}(\lambda-b(z))^{m-l}+(\lambda-b(z))^{m} \\
& =\sum_{l=1}^{m} C_{l, m}(b(y)-\lambda)^{l}(\lambda-b(y)+b(y)-b(z))^{m-l}+(\lambda-b(z))^{m} \\
& =\sum_{l=1}^{m} \sum_{h=0}^{m-l} C_{l, m, h}(b(y)-\lambda)^{l+h}(b(y)-b(z))^{m-l-h}+(\lambda-b(z))^{m} \\
& =\sum_{\gamma=0}^{m-1} C_{\gamma, m}(b(y)-\lambda)^{m-\gamma}(b(y)-b(z))^{\gamma}+(\lambda-b(z))^{m},
\end{aligned}
$$

then

$$
\begin{aligned}
\left|\left[b^{m}, \mathcal{R}^{*}\right] f(y)\right| & =\int_{R^{n}}\left|\mathcal{K}^{*}(y, z)(b(y)-b(z))^{m} f(z)\right| d z \\
& \lesssim \sum_{\gamma=0}^{m-1}|b(y)-\lambda|^{m-\gamma}\left|\left[b^{\gamma}, \mathcal{R}^{*}\right](f)(y)\right|+\left|\mathcal{R}^{*}\left((b-\lambda)^{m} f\right)(y)\right| .
\end{aligned}
$$

Let $\lambda=b_{Q}$. Then by Hölder's inequality and Lemma 2.3 we get

$$
\begin{aligned}
& \frac{1}{|Q|} \int_{Q}\left|\sum_{\gamma=0}^{m-1}\right| b(y)-\left.\lambda\right|^{m-\gamma}\left|\left[b^{\gamma}, \mathcal{R}^{*}\right](f)(y)\right| d y \\
\lesssim & \sum_{\gamma=0}^{m-1} \frac{1}{|Q|} \int_{Q}|b(y)-\lambda|^{m-\gamma}\left|\left[b^{\gamma}, \mathcal{R}^{*}\right](f)(y)\right| d y \\
\lesssim & \sum_{\gamma=0}^{m-1}\left(\frac{1}{|Q|} \int_{Q}\left|b(y)-b_{Q}\right|^{(m-\gamma) s^{\prime}} d y\right)^{1 / s^{\prime}}\left(\frac{1}{|Q|} \int_{Q}\left|\left[b^{\gamma}, \mu_{j}^{L}\right] f(y)\right|^{s} d y\right)^{1 / s}
\end{aligned}
$$




$$
\begin{aligned}
& \lesssim \sum_{\gamma=0}^{m-1}\left([b]_{\beta}^{\theta}\right)^{m-\gamma}\left(\rho\left(x_{0}\right)\right)^{\beta(m-\gamma)}\left(\frac{1}{|Q|} \int_{Q}\left|\left[b^{\gamma}, \mathcal{R}^{*}\right] f(y)\right|^{s} d y\right)^{1 / s} \\
& \lesssim \sum_{\gamma=0}^{m-1}\left([b]_{\beta}^{\theta}\right)^{m-\gamma} \inf _{x \in Q} M_{(m-\gamma) \beta, s}\left(\left[b^{\gamma}, \mathcal{R}^{*}\right] f\right)(x) .
\end{aligned}
$$

To the second term, we split $f=f_{1}+f_{2}$ with $f_{1}=f \chi_{2 Q}$. Let $p_{1}^{\prime}<\tilde{s}<s<\infty$, and $v=s \tilde{s} /(s-\tilde{s})$, by Hölder's inequality, the boundedness of $\mathcal{R}^{*}$ on $L^{\tilde{s}}\left(\mathbb{R}^{n}\right)$, and Lemma 2.3 we obtain

$$
\begin{aligned}
& \frac{1}{|Q|} \int_{Q}\left|\mathcal{R}^{*}\left(\left(b-b_{2 Q}\right)^{m} f_{1}\right)(y)\right| d y \\
\leq & \left(\frac{1}{|Q|} \int_{Q}\left|\mathcal{R}^{*}\left(\left(b-b_{Q}\right)^{m} f_{1}\right)(y)\right|^{\tilde{s}}\right)^{1 / \tilde{s}} \\
\lesssim & \left(\frac{1}{|Q|} \int_{2 Q}\left|\left(b(y)-b_{Q}\right)^{m} f(y)\right|^{\tilde{s}} d y\right)^{1 / \tilde{s}} \\
\lesssim & \left(\frac{1}{|Q|} \int_{2 Q}|f(y)|^{s} d y\right)^{1 / s}\left(\frac{1}{|Q|} \int_{2 Q}\left|b(y)-b_{Q}\right|^{m v} d y\right)^{1 / v} \\
\lesssim & \left([b]_{\beta}^{\theta}\right)^{m} \inf _{x \in Q} M_{m \beta, s} f(x) .
\end{aligned}
$$

For the remaining term, note that $\rho(y) \approx \rho\left(x_{0}\right)$ for any $y \in Q$, by Lemma 2.4 and decomposing $(2 Q)^{c}$ into annuli $2^{k} Q \backslash 2^{k-1} Q, k \geq 2$, we get

$$
\begin{aligned}
& \left|\mathcal{R}^{*}\left(\left(b-b_{Q}\right)^{m} f_{2}\right)(y)\right| \\
& \leq \int_{(2 Q)^{c}}\left|\mathcal{K}^{*}(y, z)\left(b(z)-b_{Q}\right)^{m} f(z)\right| d z \\
& \lesssim \int_{(2 Q)^{c}} \frac{\left(1+\frac{|y-z|}{\rho(y)}\right)^{-N}}{|y-z|^{n}}\left|\left(b(z)-b_{Q}\right)^{m} f(z)\right| d z \\
& +\int_{(2 Q) c} \frac{\left(1+\frac{|y-z|}{\rho(y)}\right)^{-N}}{|y-z|^{n-2}} \int_{B(z,|y-z| / 4)} \frac{V^{2}(u)}{|u-z|^{n-2}} d u\left|\left(b(z)-b_{Q}\right)^{m} f(z)\right| d z \\
& \lesssim \sum_{k \geq 2} \frac{2^{-k N}}{\left|2^{k} Q\right|} \int_{2^{k} Q}\left|\left(b(z)-b_{Q}\right)^{m} f(z)\right| d z \\
& +\sum_{k \geq 2} \frac{2^{-k N}}{\left|2^{k} Q\right|^{1-2 / n}} \int_{2^{k} Q}\left|\left(b(z)-b_{Q}\right)^{m} f(z)\right|\left|I_{2}\left(V^{2} \chi_{2^{k+2} Q}\right)(z)\right| d z,
\end{aligned}
$$

where

$$
I_{2}(f)(y)=\int_{R^{n}} \frac{f(z)}{|y-z|^{n-2}} d z
$$


is the Riesz potential. By Hölder's inequality and Lemma 2.3,

$$
\begin{aligned}
& \sum_{k \geq 2} \frac{2^{-k N}}{\left|2^{k} Q\right|} \int_{2^{k} Q}\left|\left(b(z)-b_{Q}\right)^{m} f(z)\right| d z \\
\lesssim & \sum_{k=2}^{\infty} 2^{-k N}\left(\frac{1}{\left|2^{k} Q\right|} \int_{2^{k} Q}\left|b(z)-b_{2 Q}\right|^{m s^{\prime}} d z\right)^{1 / s^{\prime}}\left(\frac{1}{\left|2^{k} Q\right|} \int_{2^{k} Q}|f(z)|^{s} d z\right)^{1 / s} \\
\lesssim & \left([b]_{\beta}^{\theta}\right)^{m} \sum_{k=2}^{\infty} 2^{-k N} \inf _{x \in Q} M_{m \beta, s}(f)(x) \\
\lesssim & \left([b]_{\beta}^{\theta}\right)^{m} \inf _{x \in Q} M_{m \beta, s}(f)(x) .
\end{aligned}
$$

By Hölder's inequality,

$$
\begin{aligned}
& \sum_{k \geq 2} \frac{2^{-k N}}{\left|2^{k} Q\right|^{1-2 / n}} \int_{2^{k} Q}\left|\left(b(z)-b_{Q}\right)^{m} f(z)\right| \mid I_{2}\left(V^{2} \chi_{2^{k+3} Q}\right)(z) d z \\
\lesssim & \sum_{k \geq 2} 2^{-k N}\left(2^{k} \rho\left(x_{0}\right)\right)^{2-n / \tilde{s}^{\prime}}\left(\left.\frac{1}{\left|2^{k} Q\right|} \int_{2^{k} Q}\left|\left(b(z)-b_{Q}\right)^{m} f(z)\right|\right|^{\tilde{s}} d z\right)^{1 / \tilde{s}}\left\|I_{2}\left(V^{2} \chi_{2^{k+3} Q}\right)\right\|_{L^{s^{\prime}}\left(\mathbb{R}^{n}\right)} .
\end{aligned}
$$

Let $v=s \tilde{s} /(s-\tilde{s})$. Then by Hölder's inequality again, we get

$$
\begin{aligned}
& \left(\frac{1}{\left|2^{k} Q\right|} \int_{2^{k} Q}\left|\left(b(z)-b_{Q}\right)^{m} f(z)\right|^{\tilde{s}} d z\right)^{1 / \tilde{s}} \\
\leq & \left(\frac{1}{\left|2^{k} Q\right|} \int_{2^{k} Q}|f(z)|^{s} d z\right)^{1 / s}\left(\frac{1}{\left|2^{k} Q\right|} \int_{2^{k} Q}\left|b(z)-b_{Q}\right|^{m v} d z\right)^{1 / v} \\
\lesssim & \left([b]_{\beta}^{\theta}\right)^{m} \inf _{x \in Q} M_{m \beta, s}(f)(x) .
\end{aligned}
$$

Since $p_{1}^{\prime}<\tilde{s}$, we have $\tilde{s}^{\prime}<p_{1}$, then $1 / \tilde{s}^{\prime}>1 / p_{1}=2 / q_{1}-2 / n$. We can choose $n / 2<\tilde{t}<$ $q_{1}$ such that $1 / \tilde{s}^{\prime}=2 / \tilde{t}-2 / n$. By $\left(L^{\tilde{t} / 2}, L^{\tilde{s}^{\prime}}\right)$-boundedness of $I_{2}, V \in R H_{\tilde{t}}$, and Lemma 2.2 , we get

$$
\begin{aligned}
& \left\|I_{2}\left(V^{2} \chi_{2^{k+3} Q}\right)\right\|_{L^{\tilde{s}^{\prime}}\left(\mathbb{R}^{n}\right)} \lesssim\left\|V^{2} \chi_{2^{k+3} Q}\right\|_{L^{\tilde{f} / 2}\left(\mathbb{R}^{n}\right)} \\
\lesssim & \left(\int_{2^{k+3} Q} V^{\tilde{t}}\right)^{2 / \tilde{t}} \lesssim\left(\frac{1}{\left|2^{k+3} Q\right|} \int_{2^{k+3} Q} V^{\tilde{t}}\right)^{2 / \tilde{t}}\left(2^{k} \rho\left(x_{0}\right)\right)^{\frac{2 n}{t}} \\
\lesssim & \left(\frac{1}{\left|2^{k+3} Q\right|} \int_{2^{k+3} Q} V\right)^{2}\left(2^{k} \rho\left(x_{0}\right)\right)^{\frac{2 n}{t}} \\
\lesssim & \left(1+\frac{2^{k} \rho\left(x_{0}\right)}{\rho\left(x_{0}\right)}\right)^{2 l_{0}}\left(2^{k} \rho\left(x_{0}\right)\right)^{\frac{2 n}{t}-4} \lesssim 2^{2 k l_{0}}\left(2^{k} \rho\left(x_{0}\right)\right)^{\frac{2 n}{t}-4} .
\end{aligned}
$$


Thus

$$
\begin{aligned}
& \sum_{k \geq 2} \frac{2^{-k N}}{\left|2^{k} Q\right|^{1-2 / n}} \int_{2^{k} Q}\left|\left(b(z)-b_{Q}\right)^{m} f(z)\right| \mid I_{2}\left(V^{2} \chi_{2^{k+3} Q}\right)(z) d z \\
& \lesssim\left([b]_{\beta}^{\theta}\right)^{m} \inf _{x \in Q} M_{m \beta, s}(f)(x) .
\end{aligned}
$$

So we get

$$
\left|\mathcal{R}^{*}\left(\left(b-b_{Q}\right)^{m} f_{2}\right)(y)\right| \lesssim\left([b]_{\beta}^{\theta}\right)^{m} \inf _{x \in Q} M_{\beta, s}(f)(x)
$$

This completes the proof of Lemma 3.1.

Lemma 3.2. Suppose $V \in R H_{q_{1}}$ with $q_{1}>n / 2$. Let $b \in \Lambda_{\beta^{\prime}}^{\theta}$ let $B=B\left(x_{0}, r\right)$ with $r \leq \gamma \rho\left(x_{0}\right)$ and let $x \in B$, then for any $y, z \in B$ we have

$$
\int_{(2 B)^{c}}\left|\mathcal{K}^{*}(y, u)-\mathcal{K}^{*}(z, u)\right|\left|b(u)-b_{B}\right|^{m}|f(u)| d u \lesssim\left([b]_{\beta}^{\theta}\right)^{m} M_{m \beta, s}(f)(x),
$$

where $s>p_{1}^{\prime}$ and $\gamma>1$.

Proof. Setting $Q=B\left(x_{0}, \gamma \rho\left(x_{0}\right)\right)$, due to the fact $\rho(y) \approx \rho(z) \approx \rho\left(x_{0}\right)$ and $|y-u| \approx$ $|z-u| \approx\left|x_{0}-u\right|$, then by Lemma 2.4 we get

$$
\int_{(2 B)^{c}}\left|\mathcal{K}^{*}(y, u)-\mathcal{K}^{*}(z, u)\right|\left|b(u)-b_{B}\right|^{m}|f(u)| d u \lesssim J_{1}+J_{2}+J_{3}+J_{4},
$$

where

$$
\begin{aligned}
& J_{1}=r^{\delta} \int_{Q \backslash 2 B} \frac{\left|f(u)\left(b(u)-b_{B}\right)^{m}\right|}{\left|x_{0}-u\right|^{n+\delta}} d u, \\
& J_{2}=r^{\delta} \rho\left(x_{0}\right)^{N} \int_{Q^{c}} \frac{\left|f(u)\left(b(u)-b_{B}\right)^{m}\right|}{\left|x_{0}-u\right|^{n+N+\delta}} d u, \\
& J_{3}=r^{\delta} \int_{Q \backslash 2 B} \frac{\left|f(u)\left(b(u)-b_{B}\right)^{m}\right|}{\left|x_{0}-u\right|^{n+\delta-2}} \int_{B\left(x_{0}, 4\left|x_{0}-u\right|\right)} \frac{V^{2}(\omega)}{|\omega-u|^{n-2}} d \omega d u, \\
& J_{4}=r^{\delta} \rho\left(x_{0}\right)^{N} \int_{Q^{c}} \frac{\left|f(u)\left(b(u)-b_{B}\right)^{m}\right|}{\left|x_{0}-u\right|^{n+\delta+N-2}} \int_{B\left(x_{0}, 4\left|x_{0}-u\right|\right)} \frac{V^{2}(\omega)}{|\omega-u|^{n-2}} d \omega d u .
\end{aligned}
$$

Let $j_{0}$ be the least integer such that $2^{j_{0}} \geq \gamma \rho\left(x_{0}\right) / r$. Splitting into annuli, we have

$$
J_{1} \leq \sum_{j=2}^{j_{0}} 2^{-\delta j} \frac{1}{\left|2^{j} B\right|} \int_{2^{j} B}|f(u)|\left|b(u)-b_{B}\right|^{m} d u .
$$


By Hölder's inequality and Lemma 2.3 we obtain for $j \leq j_{0}$

$$
\begin{aligned}
& \frac{1}{\left|2^{j} B\right|} \int_{2^{j} B}|f(u)|\left|b(u)-b_{B}\right|^{m} d u \\
\leq & \left(\frac{1}{\left|2^{j} B\right|} \int_{2^{j} B}|f(u)|^{s} d u\right)^{1 / s}\left(\frac{1}{\left|2^{j} B\right|} \int_{2^{j} B}\left|b(u)-b_{B}\right|^{m s^{\prime}} d u\right)^{1 / s^{\prime}} \\
\lesssim & \left([b]_{\beta}^{\theta}\right)^{m} M_{m \beta, s}(f)(x) .
\end{aligned}
$$

Then

$$
J_{1} \lesssim\left([b]_{\beta}^{\theta}\right)^{m} M_{m \beta, s}(f)(x)
$$

To deal with $J_{2}$, splitting into annuli and using Lemma 2.3 we have

$$
\begin{aligned}
J_{2} & \lesssim \rho\left(x_{0}\right)^{N} \sum_{j \geq j_{0}} 2^{-j \delta} \frac{1}{\left(2^{j} r\right)^{N}} \frac{1}{\left|2^{j} B\right|} \int_{2^{j} B}|f(u)|\left|b(u)-b_{B}\right|^{m} d u \\
& \lesssim\left([b]_{\beta}^{\theta}\right)^{m} \sum_{j \geq j_{0}} 2^{-j \delta}\left(\frac{2^{j} r}{\rho\left(x_{0}\right)}\right)^{-N}\left(1+\frac{2^{j} r}{\rho\left(x_{0}\right)}\right)^{m \theta^{\prime}}\left(\frac{1}{\left|2^{j} B\right|^{1-m \beta / n}} \int_{2^{j} B}|f(u)|^{s} d u\right)^{1 / s} \\
& \lesssim\left([b]_{\beta}^{\theta}\right)^{m} \sum_{j \geq j_{0}} 2^{-j \delta}\left(\frac{2^{j} r}{\rho\left(x_{0}\right)}\right)^{-\left(N-m \theta^{\prime}\right)} M_{m s \beta, s}(f)(x) .
\end{aligned}
$$

Since $\frac{2^{j} r}{\rho\left(x_{0}\right)} \geq \gamma>1$, taking $N \geq m \theta^{\prime}$, we get

$$
J_{2} \lesssim\left([b]_{\beta}^{\theta}\right)^{m} M_{m \beta, s}(f)(x)
$$

Now we consider the term $J_{3}$. Let $p_{1}^{\prime}<\tilde{s}<s$. Since $2^{j}<\gamma \rho\left(x_{0}\right) / r$ for $j<j_{0}$, then

$$
\begin{aligned}
J_{3} & \lesssim \sum_{j=2}^{j_{0}} 2^{-\delta j} \frac{1}{\left(2^{j} r\right)^{n-2}} \int_{2^{j} B}|f(u)|\left|b(u)-b_{B}\right|\left|I_{2}\left(V^{2} \chi_{B\left(x_{0}, 2^{j+2} r\right)}\right)(u)\right| d u \\
& \lesssim \sum_{j=2}^{j_{0}} 2^{-\delta j} \frac{1}{\left(2^{j} r\right)^{n-2}}\left(\int_{2^{j} B}\left|\left(b(z)-b_{B}\right) f(z)\right|^{\tilde{s}} d z\right)^{1 / \tilde{s}}\left\|I_{2}\left(V^{2} \chi_{B\left(x_{0}, 2^{j+2} r\right)}\right)\right\|_{L^{s^{\prime}}\left(\mathbb{R}^{n}\right)} \\
& \lesssim\left([b]_{\beta}^{\theta}\right)^{m} \sum_{j=2}^{j_{0}} 2^{-\delta j}\left(2^{j} r\right)^{n / s-n-2+2 n / \tilde{t}}\left(1+\frac{2^{j} r}{\rho\left(x_{0}\right)}\right)^{m \theta^{\prime}+2 l_{0}} M_{m \beta, s}(f)(x) \\
& \lesssim\left([b]_{\beta}^{\theta}\right)^{m} M_{m \beta, s}(f)(x) .
\end{aligned}
$$

Note $2^{j} r / \rho\left(x_{0}\right)>\gamma>1$, similar to the estimates for $J_{3}$ we have

$$
\begin{aligned}
J_{4} & \lesssim \sum_{j=j_{0}-1}^{\infty} 2^{-\delta j}\left(\frac{\rho\left(x_{0}\right)}{2^{j} r}\right)^{N} \frac{1}{\left(2^{j} r\right)^{n-2}} \int_{2^{j} B}|f(u)|\left|b(u)-b_{B}\right|^{m}\left|I_{2}\left(V^{2} \chi_{B\left(x_{0}, 2^{j+2} r\right)}\right)(u)\right| d u \\
& \lesssim\left([b]_{\beta}^{\theta}\right)^{m} \sum_{j=j_{0}-1}^{\infty} 2^{-\delta j}\left(\frac{2^{j} r}{\rho\left(x_{0}\right)}\right)^{-\left(N-m \theta^{\prime}-2 l_{0}\right)} M_{m \beta, s}(f)(x) .
\end{aligned}
$$


Then, taking $N>m \theta^{\prime}+2 l_{0}$ we get

$$
J_{4} \lesssim\left([b]_{\beta}^{\theta}\right)^{m} M_{m \beta, s}(f)(x) .
$$

Thus, we complete the proof.

Lemma 3.3. Let $p_{1}^{\prime}<s<\infty$, let $B=B\left(x_{0}, r\right)$ with $r \leq \gamma \rho\left(x_{0}\right)$ and let $x \in B$. Then

$$
M_{\rho, \gamma}^{\sharp}\left(\left[b^{m}, \mathcal{R}^{*}\right] f\right)(x) \lesssim\left([b]_{\beta}^{\theta}\right)^{m}\left(M_{m \beta, s}(f)(x)+M_{m \beta, s}\left(\mathcal{R}^{*} f\right)(x)\right) .
$$

Proof. Write

$$
\begin{aligned}
& \frac{1}{|B|} \int_{B}\left|\left[b^{m}, \mathcal{R}^{*}\right] f(y)-\left(\left[b^{m}, \mathcal{R}^{*}\right] f\right)_{B}\right| d y \\
\leq & \frac{2}{|B|} \int_{B}\left|\left(b(y)-b_{B}\right)^{m} R^{*} f(y)\right| d y+\frac{2}{|B|} \int_{B}\left|\mathcal{R}^{*}\left(\left(b-b_{B}\right)^{m} f_{1}\right)(y)\right| d y \\
& +\frac{1}{|B|} \int_{B}\left|\mathcal{R}^{*}\left(\left(b-b_{B}\right)^{m} f_{2}\right)(y)-\left(\mathcal{R}^{*}\left(\left(b-b_{B}\right)^{m} f_{2}\right)\right)_{B}\right| d y \\
= & K_{1}+K_{2}+K_{3},
\end{aligned}
$$

where $f=f_{1}+f_{2}$ with $f_{1}=f \chi_{2 B}$.

Since $r \leq \gamma \rho\left(x_{0}\right)$ and $\rho(x) \approx \rho\left(x_{0}\right)$, by Hölder's inequality and Lemma 2.3, we get

$$
\begin{aligned}
K_{1} & \leq\left(\frac{1}{|B|} \int_{B}\left|b(y)-b_{B}\right|^{m s^{\prime}} d y\right)^{1 / s^{\prime}}\left(\frac{1}{|B|} \int_{B}\left|\mathcal{R}^{*} f(y)\right|^{s} d y\right)^{1 / s} \\
& \lesssim\left([b]_{\beta}^{\theta}\right)^{m} r^{m \beta}\left(\frac{1}{|B|} \int_{B}\left|\mathcal{R}^{*} f(y)\right|^{s} d y\right)^{1 / s} \lesssim\left([b]_{\beta}^{\theta}\right)^{m} M_{m \beta, s}\left(\mathcal{R}^{*} f\right)(x) .
\end{aligned}
$$

Select $\tilde{s}$ so that $p_{0}^{\prime}<\tilde{s}<s$. Then by the $L^{p}$-boundedness of $\mathcal{R}^{*}$ and Lemma 2.3,

$$
\begin{aligned}
K_{2} & \lesssim\left(\frac{1}{|B|} \int_{B}\left|\mathcal{R}^{*}\left(\left(b-b_{B}\right)^{m} f_{1}\right)(y)\right|^{\tilde{s}} d y\right)^{1 / \tilde{s}} \\
& \lesssim\left(\frac{1}{|B|} \int_{2 B}\left|\left(b(y)-b_{B}\right)^{f}(y)\right|^{\tilde{s}} d y\right)^{1 / \tilde{s}} \\
& \lesssim\left([b]_{\beta}^{\theta}\right)^{m} M_{m \beta, s}\left(\mathcal{R}^{*} f\right)(x) .
\end{aligned}
$$

By Lemma 3.2,

$$
\begin{aligned}
K_{3} & \leq \frac{1}{|B|^{2}} \int_{B} \int_{B} \int_{(2 B)^{c}}\left|\mathcal{K}^{*}(y, u)-\mathcal{K}^{*}(z, u)\right|\left|b(u)-b_{B}\right|^{m}|f(u)| d u d z d y \\
& \lesssim \int_{(2 B)^{c}}\left|\mathcal{K}^{*}(y, u)-\mathcal{K}^{*}(z, u)\right|\left|b(u)-b_{B}\right|^{m}|f(u)| d u \\
& \lesssim\left([b]_{\beta}^{\theta}\right)^{m} M_{m \beta, s}\left(\mathcal{R}^{*} f\right)(x) .
\end{aligned}
$$

So, we complete the proof. 
Now let us prove Theorem 1.1.

Choose numbers $t_{\alpha}$ such that $\frac{1}{t_{\alpha}}=\frac{1}{p}-\frac{\alpha \beta}{n}, \alpha=0,1, \cdots, m-1$. Then $\frac{1}{q}=\frac{1}{t_{\alpha}}-\frac{(m-\alpha) \beta}{n}$. We need to prove the following inequality

$$
\left\|\left[b^{m}, \mathcal{R}^{*}\right] f\right\|_{L^{q}\left(\mathbb{R}^{n}\right)}^{q} \lesssim\left([b]_{\beta}^{\theta}\right)^{m q}\|f\|_{L^{p}\left(\mathbb{R}^{n}\right)}^{q}+\sum_{\alpha=0}^{m-1}\left([b]_{\beta}^{\theta}\right)^{(m-\alpha) q}\left\|\left[b^{\alpha}, \mathcal{R}^{*}\right](f)\right\|_{L^{t_{\alpha}}\left(\mathbb{R}^{n}\right)}^{q} .
$$

If (3.1) holds, then Theorem 1.1 will be proved by the mathematical induction. In fact, when $m=1$, we have $\alpha=0$ and $p=t_{\alpha}$. Note that $\left[b^{0}, \mathcal{R}^{*}\right]=\mathcal{R}^{*}$, by the boundedness of $\mathcal{R}^{*}$ on $L^{p}\left(\mathbb{R}^{n}\right)$ for $p_{1}^{\prime}<p<\infty$, then $\left[b, \mathcal{R}^{*}\right]$ is bounded from $L^{p}\left(\mathbb{R}^{n}\right)$ into $L^{q}\left(\mathbb{R}^{n}\right)$. Suppose that the $\left(L^{p}, L^{t_{\alpha}}\right)$ - boundedness of $\left[b^{\alpha}, \mathcal{R}^{*}\right]$ hold for $\frac{1}{t_{\alpha}}=\frac{1}{p}-\frac{\alpha \beta}{n}$, that is

$$
\left\|\left[b^{\alpha}, \mathcal{R}^{*}\right](f)\right\|_{L^{t_{\alpha}\left(\mathbb{R}^{n}\right)}} \lesssim\left([b]_{\beta}^{\theta}\right)^{\alpha}\|f\|_{L^{p}\left(\mathbb{R}^{n}\right)}
$$

where $\alpha=2,3, \cdots, m-1$, then by (3.1) we get

$$
\left\|\left[b^{m}, \mathcal{R}^{*}\right](f)\right\|_{L^{q}\left(\mathbb{R}^{n}\right)} \lesssim\left([b]_{\beta}^{\theta}\right)^{m}\|f\|_{L^{p}\left(\mathbb{R}^{n}\right)} .
$$

In the following, we will focus on the proof of (3.1).

Let $p_{1}^{\prime}<s<p<\infty, f \in L^{p}\left(\mathbb{R}^{n}\right)$. By Proposition 2.3 we have

$$
\begin{aligned}
& \left\|\left[b^{m}, \mathcal{R}^{*}\right] f\right\|_{L^{q}\left(\mathbb{R}^{n}\right)}^{q} \leq \int_{\mathbb{R}^{n}}\left|M_{\rho, \delta}\left(\left[b^{m}, \mathcal{R}^{*}\right] f\right)(x)\right|^{q} d x \\
\leq & \int_{\mathbb{R}^{n}}\left|M_{\rho, \gamma}^{\sharp}\left(\left[b^{m}, \mathcal{R}^{*}\right] f\right)(x)\right|^{q} d x+\sum_{k}\left|Q_{k}\right|\left(\frac{1}{\left|Q_{k}\right|} \int_{2 Q_{k}}\left|\left[b^{m}, \mathcal{R}^{*}\right] f(x)\right| d x\right)^{q} .
\end{aligned}
$$

By Lemma 3.3,

$$
\begin{aligned}
& M_{\rho, \gamma}^{\sharp}\left(\left[b^{m}, \mathcal{R}^{*}\right] f\right)(x) \\
\lesssim & \left([b]_{\beta}^{\theta}\right)^{m} M_{m \beta, s}(f)(x)+\sum_{\alpha=0}^{m-1}\left([b]_{\beta}^{\theta}\right)^{m-\alpha} M_{(m-\alpha) \beta, s}\left(\left[b^{\alpha}, \mathcal{R}^{*}\right] f\right)(x) .
\end{aligned}
$$

Since

and $t_{\alpha}=p$ when $\alpha=0$, then

$$
\frac{1}{q}=\frac{1}{t_{\alpha}}-\frac{(m-\alpha) \beta}{n}
$$

$$
\begin{aligned}
& \int_{\mathbb{R}^{n}}\left|M_{\rho, \gamma}^{\sharp}\left(\left[b^{m}, \mathcal{R}^{*}\right] f\right)(x)\right|^{q} d x \\
& \lesssim\left([b]_{\beta}^{\theta}\right)^{m q} \int_{\mathbb{R}^{n}}\left|M_{m \beta, s}(f)(x)\right|^{q} d x \\
&+\sum_{\alpha=0}^{m-1}\left([b]_{\beta}^{\theta}\right)^{(m-\alpha) q} \int_{\mathbb{R}^{n}}\left|M_{(m-\alpha) \beta, s}\left(\left[b^{\alpha}, \mathcal{R}^{*}\right] f\right)(x)\right|^{q} d x \\
& \lesssim\left([b]_{\beta}^{\theta}\right)^{m q}\|f\|_{L^{p}\left(\mathbb{R}^{n}\right)}^{q}+\sum_{\alpha=0}^{m-1}\left([b]_{\beta}^{\theta}\right)^{(m-\alpha) q}\left\|\left[b^{\alpha}, \mathcal{R}^{*}\right](f)\right\|_{L^{t_{\alpha}}\left(\mathbb{R}^{n}\right)^{\cdot}}^{q}
\end{aligned}
$$


By Proposition 2.2 and Lemma 3.1 we have

$$
\begin{aligned}
& \sum_{k}\left|Q_{k}\right|\left(\frac{1}{\left|Q_{k}\right|} \int_{2 Q_{k}}\left|\left[b^{m}, \mathcal{R}^{*}\right] f(x)\right| d x\right)^{q} \\
\lesssim & \left([b]_{\beta}^{\theta}\right)^{m q} \sum_{k} \int_{2 Q_{k}}\left|M_{m \beta, s}(f)\right|^{q} d x \\
& \quad+\sum_{\alpha=0}^{m-1}\left([b]_{\beta}^{\theta}\right)^{(m-\alpha) p} \sum_{k} \int_{2 Q_{k}}\left|M_{(m-\alpha) \beta, s}\left(\left[b^{\alpha}, \mathcal{R}^{*}\right] f\right)\right|^{q} d x \\
\lesssim & \left([b]_{\beta}^{\theta}\right)^{m q} \int_{\mathbb{R}^{n}}\left|M_{m \beta, s}(f)(x)\right|^{q} d x \\
& \quad+\sum_{\alpha=0}^{m-1}\left([b]_{\beta}^{\theta}\right)^{(m-\alpha) q} \int_{\mathbb{R}^{n}}\left|M_{(m-\alpha) \beta, s}\left(\left[b^{\alpha}, \mathcal{R}^{*}\right] f\right)(x)\right|^{q} d x \\
\lesssim & \left([b]_{\beta}^{\theta}\right)^{m q}\|f\|_{L^{p}\left(\mathbb{R}^{n}\right)}^{q}+\sum_{\alpha=0}^{m-1}\left([b]_{\beta}^{\theta}\right)^{(m-\alpha) q}\left\|\left[b^{\alpha}, \mathcal{R}^{*}\right](f)\right\|_{L^{t_{\alpha}}\left(\mathbb{R}^{n}\right)}^{q} .
\end{aligned}
$$

Then the proof of (3.1) is finished.

\section{References}

[1] Y. Liu and J. Dong, Some estimates of higher order Riesz transform related to Schrödinger type operators, Potential Anal., 32 (2010), 41-55.

[2] Z. Shen, $L^{p}$ estimates for Schrödinger operators with certain potentials, Ann. Inst. Fourier, 45 (1995), 513-546.

[3] Y. Liu and J. Sheng, Some estimates for commutators of Riesz transforms associated with Schrödinger operators, J. Math. Anal. Appl., 419 (2014), 298-328.

[4] B. Bongioanni, E. Harboure and O. Salinas, Commutators of Riesz transforms related to Schrödinger operators, J. Fourier Anal. Appl., 17 (2011), 115-134.

[5] Y. Xia and M. Chen, Hardy type estimates for commutators of fractional integrals associated with Schrödinger, J. Nonlinear Sci. Appl., 10 (2017), 3155-3167.

[6] J. Dziubański and J. Zienkiewicz, Hardy space $H^{1}$ associated to Schrödinger operator with potential satisfying reverse Hölder inequality, Rev. Mat. Iberoamericana, 15 (1999), 279-296.

[7] J. Dziubański and J. Zienkiewicz, $H^{p}$ spaces associated with Schrödinger operators with potentials from reverse Hölder classes, Colloq. Math., 98 (2003), 5-38.

[8] Y. Liu, J. Zhang, J. Sheng and L. Wang, Some estimates for commutators of Riesz transform associated with Schrödinger operators, Czechoslovak Math. J., 66 (2016), 169-191. 